PROCEEDINGS OF THE

AMERICAN MATHEMATICAL SOCIETY

Volume 133, Number 4, Pages 1135-1142

S 0002-9939(04)07615-4

Article electronically published on October 15, 2004

\title{
MULTIPLICATIVELY SPECTRUM-PRESERVING MAPS OF FUNCTION ALGEBRAS
}

\author{
N. V. RAO AND A. K. ROY
}

(Communicated by N. Tomczak-Jaegermann)

\begin{abstract}
Let $X$ be a compact Hausdorff space and $\mathcal{A} \subset C(X)$ a function algebra. Assume that $X$ is the maximal ideal space of $\mathcal{A}$. Denoting by $\sigma(f)$ the spectrum of an $f \in \mathcal{A}$, which in this case coincides with the range of $f$, a result of Molnár is generalized by our Main Theorem: If $\Phi: \mathcal{A} \rightarrow \mathcal{A}$ is a surjective map with the property $\sigma(f g)=\sigma(\Phi(f) \Phi(g))$ for every pair of functions $f, g \in \mathcal{A}$, then there exists a homeomorphism $\Lambda: X \rightarrow X$ such that

$$
\Phi(f)(\Lambda(x))=\tau(x) f(x)
$$
\end{abstract}

for every $x \in X$ and every $f \in \mathcal{A}$ with $\tau=\Phi(1)$.

\section{INTRODUCTION}

Molnár [M, Theorem 5] proved the following theorem: If $X$ is a first-countable compact Hausdorff space and $C(X)$, the algebra of complex-valued continuous functions on $X$, and

$$
\Phi: C(X) \rightarrow C(X)
$$

a surjective mapping such that

$$
\text { for every pair of functions } f, g \in C(X), \sigma(f g)=\sigma(\Phi(f) \Phi(g))
$$

where $\sigma(f)$ denotes the spectrum of $f$, which in this case would be simply $f(X)$, the range of $f$, then there exists a homeomorphism $\varphi$ of $X$ onto itself and a function $\tau$, whose range is $\{-1,1\}$ such that

$$
\Phi(f)(x)=\tau(x) f(\varphi(x)) \text { for all } x \in X \text { and all } f \in C(X) .
$$

In this paper we deal with a function algebra $\mathcal{A}$ in place of $C(X)$ and regard $X$ as the maximal ideal space of $\mathcal{A}$. $X$ is of course compact Hausdorff but not necessarily first-countable. For this purpose, we need to recall some results of Bishop and de Leeuw $\mathrm{BL}$ concerning function algebras, peaking functions, generalized peak points etc., for which a readable exposition may be found in $[\mathrm{Br}$, Chapter 2] and [P, Chapter 8].

1.1 Peaking function. A function $f$ in $\mathcal{A}$ is said to be a peaking function if for any $x$ in $X$, either $f(x)=1$ or $|f(x)|<1$ and the set $\{x: x \in X, f(x)=1\}$, denoted by $P(f)$ and referred to as the peaking set, is non-empty.

Received by the editors January 21, 2003 and, in revised form, April 2, 2003 and December 2, 2003.

2000 Mathematics Subject Classification. Primary 46J10, 46J20.

Key words and phrases. Automorphism, function algebra, spectrum, boundaries. 
1.2. Generalized peak point. A point $x$ in $X$ is said to be a generalized peak point for the algebra $\mathcal{A}$ if, given any neighborhood $V$ of $x$, there exists a peaking function $f$ in $\mathcal{A}$ such that $P(f) \subset V, f(x)=1$.

The set of all generalized peak points is called the Choquet boundary of $\mathcal{A}$ and denoted by $\partial_{\mathcal{A}}(X)$. Its closure is the so-called Shilov boundary of $\mathcal{A}$. Since any $f \in \mathcal{A}$ assumes its maximum modulus $\|f\|_{\infty}:=\sup _{x \in X}|f(x)|$ on the Choquet boundary (see [P, Prop. 6.3]), we see that

$$
\text { any peaking set meets } \partial_{\mathcal{A}}(X) \text {. }
$$

Also, given any $x \in X$, there exists a probability measure $\mu$, a representing measure for $x$, supported on the Shilov boundary $S=\overline{\partial_{\mathcal{A}}(X)}$ such that for every $f \in \mathcal{A}$,

$$
f(x)=\int_{S} f d \mu .
$$

The following theorem will be invoked several times in the proof of our Main Theorem in the next section.

1.5. Theorem (Bishop). Given any peaking set $E$ and any $f \in \mathcal{A}$, there exists a peaking function $h$ in $\mathcal{A}$ with $P(h)=E$ and $|f(z) h(z)|<\max _{E}|f|$ for any $z \notin E$.

A proof may be found in $[\mathrm{Br}$, page 102]. At one point in the next section, we shall need the fact contained in the following proposition.

1.6. Proposition. Any family of peaking sets $E_{\alpha}$, with finite intersection property, has a common intersection with $\partial_{\mathcal{A}}(X)$.

Proof. The proof is a convexity argument. Let $S_{\mathcal{A}}=\left\{L \in \mathcal{A}^{*}:\|L\|=L(1)=1\right\}$ be the state space of $\mathcal{A}$. We know that (see $\left[\mathrm{P}\right.$ page 37]) $\varphi\left(\partial_{\mathcal{A}}(X)\right)=\operatorname{ext}\left(S_{\mathcal{A}}\right)$ where $\operatorname{ext}\left(S_{\mathcal{A}}\right)$ denotes the set of extreme points of the compact convex set $S_{\mathcal{A}} \subset$ $\mathcal{A}^{*}$, non-empty by the Krein-Milman theorem, and $\varphi$ denotes the evaluation map $x \rightsquigarrow \varphi(x)$ that imbeds $X$ homeomorphically into $S_{\mathcal{A}}$ with weak* topology. Each $F_{\alpha}:=$ weak $^{*}$ closed convex hull of $\varphi\left(E_{\alpha}\right)$, where $E_{\alpha}=\left\{x \in X: h_{\alpha}(x)=1\right\}$ and each $h_{\alpha} \in \mathcal{A}$ is the associated peaking function, is a weak* closed face of $S_{\mathcal{A}}$ - in fact, $F_{\alpha}=\left\{L \in S_{\mathcal{A}}: L\left(h_{\alpha}\right)=1\right\}$. Consequently by the finite intersection property, $F:=\bigcap_{\alpha} F_{\alpha}$ is a non-empty weak ${ }^{*}$ closed face of $S_{\mathcal{A}}$ and therefore has an extreme point $p$ that necessarily belongs to $\operatorname{ext}\left(S_{\mathcal{A}}\right)$ and is therefore of the form $\varphi(x)$ for some $x \in \partial_{\mathcal{A}}(X)$. But $p \in \operatorname{ext}\left(F_{\alpha}\right) \subset \varphi\left(E_{\alpha}\right)$ for every $\alpha$ by the Milman theorem; hence $x \in \bigcap_{\alpha} E_{\alpha}$, and we are done.

\section{Proof of the Main Theorem}

In the sequel $f, g, h, k$, etc. denote functions from $\mathcal{A}$ and $c$ denotes a generic constant. Also for any $f \in \mathcal{A}$, we shall sometimes abbreviate $\|f\|_{\infty}$ to $\|f\|$. It is convenient to present the proof of our theorem as a sequence of remarks. We point out that the proofs of these remarks, though modelled in several instances on [M], are rendered somewhat complicated by the more general situation that is being considered here.

Remark 1. Reduction. Since $\sigma\left(1^{2}\right)=\sigma\left(\Phi(1)^{2}\right)$, we have $\Phi(1)^{2}=1$, and so by defining $\Psi f=\Phi(1) \Phi(f)$, we see that $\Psi(1)=(\Phi(1))^{2}=1$. Furthermore, $\Psi(f) \Psi(g)=\Phi(1) \Phi(f) \Phi(1) \Phi(g)=\Phi(f) \Phi(g)$ and, consequently,

$$
\sigma(f g)=\sigma(\Psi(f) \Psi(g)) .
$$


Now if we prove the existence of a homeomorphic self-map $\Lambda$ of $X$ such that

$$
\Psi(f)(\Lambda(x))=f(x)
$$

for every $x \in X$, we would have proved the theorem mentioned in the abstract. So from now on, we assume that $\Phi(1)=1$ and so

$$
\sigma(f)=\sigma(\Phi(f)) \quad \forall f \in \mathcal{A},
$$

from which it immediately follows that

$$
\|f\|_{\infty}=\|\Phi(f)\|_{\infty} .
$$

Remark 2. If $f, g \in \mathcal{A}$, then $|f| \leq|g|$ on $\partial_{\mathcal{A}}(X)$ if and only if

$$
\text { for every } c \geq 0 \text { and every } h,|g h| \leq c \text { implies }|f h| \leq c \text {. }
$$

Proof. That $|f| \leq|g|$ on $\partial_{\mathcal{A}}(X)$ implies (2.3) is obvious by (1.4). Assume that (2.3) is true but $|f| \not \leq|g|$ on $\partial_{\mathcal{A}}(X)$. Hence there must exist an $x_{0}$ in $\partial_{\mathcal{A}}(X)$ such that

$$
\left|f\left(x_{0}\right)\right|>\left|g\left(x_{0}\right)\right|
$$

for, otherwise, $|f| \leq|g|$ on $\partial_{\mathcal{A}}(X)$.

Let $\gamma=\frac{1}{2}\left(\left|f\left(x_{0}\right)\right|+\left|g\left(x_{0}\right)\right|\right)$. So $\left|g\left(x_{0}\right)\right|<\gamma<\left|f\left(x_{0}\right)\right|$, and there exists an open neighborhood $V$ of $x_{0}$ such that $|g(x)|<\gamma$ in $V$ and a function $h$ such that $h\left(x_{0}\right)=$ $1=\|h\|$, and $|g(x) h(x)|<\gamma$ in $X \backslash V$. Such an $h$ exists, because $x_{0}$ is a generalized peak point for $\mathcal{A}$. Therefore $|g h|<\gamma$ on all of $X$, but $\left|f\left(x_{0}\right) h\left(x_{0}\right)\right|=\left|f\left(x_{0}\right)\right|>\gamma$, a contradiction. This proves the assertion (2.3).

From (2.3), we can deduce the following:

$$
\text { if } \sigma(f h)=\sigma(g h) \text { for every } h, \text { then on } \partial_{\mathcal{A}}(X),|f|=|g| \text {. }
$$

Since $\sigma(f h)=\sigma(g h) \forall h \in \mathcal{A}$ we see that for any constant $c \geq 0$ and any $h \in \mathcal{A}$, $|g h| \leq c$ implies $|f h| \leq c$ and so (2.3) gives $|f| \leq|g|$ on $\partial_{\mathcal{A}}(X)$. Since the hypothesis is symmetric in $f, g$, we obtain also $|g| \leq|f|$ on $\partial_{\mathcal{A}}(X)$. Combining, we have (2.4).

As a consequence we have

Remark 3.

$$
\text { On } \partial_{\mathcal{A}}(X),|f| \leq|g| \Leftrightarrow|\Phi(f)| \leq|\Phi(g)| \forall f, g \in \mathcal{A} \text {. }
$$

Proof. Assume that $|f| \leq|g|$ on $\partial_{\mathcal{A}}(X)$ and $|\Phi(g) k| \leq c$ for some $k \in \mathcal{A}$ and $c \geq 0$. $\Phi$ being surjective, there exists an $h \in \mathcal{A}$ such that $\Phi(h)=k$. Hence we have

$$
|\Phi(g) \Phi(h)| \leq c .
$$

But since

$$
\sigma(g h)=\sigma(\Phi(g) \Phi(h)),
$$

we obtain $|g h| \leq c$ and so by $(2.3),|f h| \leq c$. Since

$$
\sigma(f h)=\sigma(\Phi(f) \Phi(h)),
$$

we obtain $|\Phi(f) \Phi(h)|=|\Phi(f) k| \leq c$. Now since $k, c$ are arbitrary, from Remark 2, it follows that

$$
|\Phi(f)| \leq|\Phi(g)| \text { on } \partial_{\mathcal{A}}(X) .
$$

Now the other implication has a similar proof. 
Remark 4. For any fixed $x \in \partial_{\mathcal{A}}(X)$,

$$
E:=\bigcap_{f \in \mathcal{F}_{x}} P(f)=\{x\},
$$

where $\mathcal{F}_{x}$ denotes the family of all peaking functions $f \in A$ such that $f(x)=1$.

Proof. Assume $E$ contains a point $y$ other than $x$. From (1.2) it follows that every point of $\partial_{\mathcal{A}}(X)$ is a generalized peak point for $\mathcal{A}$, which means that, given any neighborhood $V$ of $x$, there exists a peaking function $h$ in $\mathcal{A}$ such that $h(x)=1=$ $\|h\|$ and $|h|<1$ outside $V$, which means $P(h) \subset V$. So if we choose a neighborhood $V$ of $x$ that does not contain $y$, since $E \subset V, y \notin E$, a contradiction.

We now have the important

Remark 5. If $x \in \partial_{\mathcal{A}}(X)$,

$$
\bigcap_{f \in \mathcal{F}_{x}} P(\Phi(f)) \text { contains one and only one generalized peak point. }
$$

First, because of $(2.1), \Phi(f)$ is a peaking function if and only if $f$ is a peaking function. Also, each $P(\Phi(f))$ is compact.

Secondly, if $f_{1}, f_{2}, \ldots, f_{n}$ belong to $\mathcal{F}_{x}$, then $g=f_{1} f_{2} \ldots f_{n}$ belongs to $\mathcal{F}_{x}$. Since $|g| \leq\left|f_{i}\right|$, we obtain in view of $(2.5)$,

$$
|\Phi(g)| \leq\left|\Phi\left(f_{i}\right)\right| \text { for each } 1 \leq i \leq n \text { on } \partial_{\mathcal{A}}(X) .
$$

Since $g$ is a peaking function, so is $\Phi(g)$, and so $\Phi(g)(\xi)=1$ for some $\xi$ in $\partial_{\mathcal{A}}(X)$. Then $\Phi\left(f_{i}\right)(\xi)=1$ for $1 \leq i \leq n$ or

$$
\bigcap_{1 \leq i \leq n} P\left(\Phi\left(f_{i}\right)\right) \neq \emptyset
$$

This proves that the family of sets $\left\{P(\Phi(f)): f \in \mathcal{F}_{x}\right\}$ has the finite intersection property, and since each of them is compact, it must be that

$$
E^{\prime}=\bigcap_{f \in \mathcal{F}_{x}} P(\Phi(f)) \neq \emptyset .
$$

Thus, $E^{\prime}$ being a non-empty intersection of peaking sets must intersect $\partial_{\mathcal{A}}(X)$ by Proposition 1.6.

Thirdly, if $y \in E^{\prime} \cap \partial_{\mathcal{A}}(X)$, let $k$ be a peaking function such that $k(y)=1$. By surjectivity of $\Phi, k=\Phi(h)$ for some peaking function $h \in \mathcal{A}$ (recall that $\sigma(k)=\sigma(h))$. We claim that $h(x)=1$. To show this, choose any neighborhood $V$ of $x$ and a peaking function $g$ such that $g(x)=1$ and $|g|<1$ outside $V$. So $g \in \mathcal{F}_{x}$ and hence $\Phi(g)(y)=1$. Consider $\Phi(g) \Phi(h)=\lambda \in \mathcal{A}$. $\Phi(g), \Phi(h)$ being both peaking functions that take the value 1 at $y$, we see that $\lambda(y)=1$ and $\lambda$ is a peaking function. Again $\Phi$ being surjective, there exists a peaking function $\mu \in \mathcal{A}$ such that $\Phi(\mu)=\lambda$. Since $|\lambda| \leq|\Phi(g)| \wedge|\Phi(h)|$ on $\partial_{\mathcal{A}}(X)$, by (2.5) it follows that $|\mu| \leq|g| \wedge|h|$ on $\partial_{\mathcal{A}}(X)$. Hence there exists a $\xi \in \partial_{\mathcal{A}}(X)$ such that $\mu(\xi)=1$, and so $g(\xi)=h(\xi)=1$, which implies that $\xi \in V$. Since $V$ is an arbitrary neighborhood of $x$ and $h$ is continuous, we get

$$
h(x)=1 .
$$

Lastly, if there is a generalized peak point $z$ other than $y$ in $E^{\prime}$, we can choose $k$ in such a way that $k(y)=1,|k(z)|<1$. $\Phi$ being surjective, we obtain $h^{\prime}$ such that $\Phi\left(h^{\prime}\right)=k$. So by the previous paragraph, we see that $h^{\prime}$ belongs to $\mathcal{F}_{x}$ and so 
$\Phi\left(h^{\prime}\right)=1$ on $E^{\prime}$ and consequently $k(z)=1$, which is a contradiction. This proves Remark 5.

Let the unique point $y$ given by Remark 5 be denoted by $\tau(x)$ since it depends on $x$ and nothing else. We sum up what we established above as follows:

Remark 6. If $x \in \partial_{\mathcal{A}}(X)$ and $f \in \mathcal{F}_{x}$, then $\tau(x) \in \partial_{\mathcal{A}}(X)$ and $\Phi(f)$ belongs to $\mathcal{F}_{\tau(x)}$. Conversely, if $k \in \mathcal{F}_{\tau(x)}$ and $\Phi(h)=k$, then $h \in \mathcal{F}_{x}$.

We now have

Remark 7. $\Phi$ is injective and homogeneous, i.e., $\Phi(c f)=c \Phi(f)$ for any $f \in \mathcal{A}$ and $c \in \mathbb{C}$.

Proof. Suppose if possible that $\Phi(f)=\Phi(g)$ for some $f \neq g$. For any $h \in \mathcal{A}$, $\Phi(f) \Phi(h)=\Phi(g) \Phi(h)$ and consequently,

$$
\sigma(\Phi(f) \Phi(h))=\sigma(\Phi(g) \Phi(h)),
$$

from which we see that

$$
\sigma(f h)=\sigma(g h)
$$

We deduce from (2.4) that $|f|=|g|$ on $\partial_{\mathcal{A}}(X)$. Since $f \neq g$, there exists a $y \in$ $\partial_{\mathcal{A}}(X)$ such that $f(y) \neq g(y)$; for otherwise $f-g$ would vanish on $\partial_{\mathcal{A}}(X)$, and so $f=g$ on $X$ by (1.4). We may assume that $f(y) \neq 0$ because if $f(y)=0$, then, since $|f(y)|=|g(y)|$, it would follow that $g(y)=0=f(y)$. Therefore we can choose a neighborhood $V$ of $y$ and a peaking function $h$ such that $1=h(y),|h(z)|<1$ outside $V$. Then $E:=P(h) \subset V$. By (1.5), we can modify $h$ so that it would still be a peaking function that peaks on $E$ and moreover satisfies the following:

$$
\begin{array}{r}
|f(z) h(z)|<\max _{E}|f|=\max _{X}|f h|, \\
\left|g(z) h(z)<\max _{E}\right| g\left|=\max _{X}\right| g h \mid
\end{array}
$$

for all $z$ outside $E$.

There exists $\xi \in E$ such that $|f(\xi)|=\max _{E}|f|=\|f h\|_{\infty}$. Since $\sigma(f h)=\sigma(g h)$, $f(\xi)=f(\xi) h(\xi)=g(z) h(z)$ for some $z \in X$. If $z \notin E$, then $|g(z) h(z)|<\max _{E}|g|=$ $\|g h\|_{\infty}=\|f h\|_{\infty}=|f(\xi)|$, a contradiction. So $z \in E$ and $f(\xi)=g(z)$ where both $\xi, z$ lie in $V$. Since $V$ is an arbitrary neighborhood of $y$ and $f, g$ are continuous, we get $f(y)=g(y)$, again a contradiction.

Thus

$$
\sigma(f h)=\sigma(g h) \forall h \Leftrightarrow f=g
$$

and $\Phi$ is injective.

Now for the homogeneity. Notice that

$$
\sigma(\Phi(c f) \Phi(h))=\sigma(c f h)=c \sigma(f h)=c \sigma(\Phi(f) \Phi(h))=\sigma(c \Phi(f) \Phi(h)) .
$$

Since $\Phi$ is bijective, we see that $\Phi(c f)=c \Phi(f) \forall f \in \mathcal{A}$.

Remark 8.

$$
|f(x)|=\mid \Phi(f)(\tau(x))) \mid \quad \forall f \in \mathcal{A}, \quad \forall x \in \partial_{\mathcal{A}}(X) .
$$

Proof. Take $f \in \mathcal{A}$ and assume first that $f(y) \neq 0, y \in \partial_{\mathcal{A}}(X)$. In this case, for any given neighborhood $V$ of $y$, we can find a function $h$ such that $h(y)=1=\|h\|$ and $f h$ attains its maximum modulus in $V$. (To find $h$, let $k$ be a peaking function 
with $P(k) \subset V$, and let $h=k^{n}$ for some sufficiently large positive integer $n$.) There exists a $\xi$ in $V$ such that

$$
|f(\xi) h(\xi)|=\|f h\|_{\infty} .
$$

But $\sigma(\Phi(f) \Phi(h))=\sigma(f h)$ from which it follows that $|\Phi(f(\tau(y))) \Phi(h(\tau(y)))| \leq$ $|f(\xi) h(\xi)|$. Since $\Phi(h(\tau(y)))=h(y)=1$ (Remark 6),

$|h(\xi)| \leq 1$, we get

$$
\mid \Phi(f)(\tau(y)))|\leq| f(\xi) \mid .
$$

$V$ being arbitrary and $f$ continuous, we have

$$
\mid \Phi(f)(\tau(y)))|\leq| f(y) \mid .
$$

If, on the other hand, $f(y)=0$, we could ensure that $h$ satisfies $h(y)=1=$ $\|h\|_{\infty}$ and $\|f h\|_{\infty}<\epsilon$ for some preassigned $\epsilon>0$. Hence once again because $\sigma(\Phi(f) \Phi(h))=\sigma(f h)$, we see that $\|\Phi(f) \Phi(h)\|<\epsilon$ by $(2.2)$ and so

$$
|\Phi(f)(\tau(y)) \Phi(h)(\tau(y))|<\epsilon,
$$

and since $\Phi(h)(\tau(y))=1$, we get

$$
|\Phi(f)(\tau(y))|<\epsilon,
$$

which proves $f(y)=\Phi(f)(\tau(y))=0$.

Now let $V$ be any neighborhood of $\tau(y)$, and assume that $\Phi(f)(\tau(y)) \neq 0$. We can, as before, choose $h^{\prime}$ with $h^{\prime}(\tau(y))=1=\left\|h^{\prime}\right\|$ and $\Phi(f) h^{\prime}$ attains its maximum modulus at a point $\xi$ in $V$. Since $\Phi$ is surjective, let $\Phi(h)=h^{\prime}$. By Remark 6 , $h(y)=1$ and since $f(y) h(y)$ belongs to $\sigma(f h)=\sigma(\Phi(f) \Phi(h))$, we get

$$
f(y)=\Phi(f)\left(\xi^{\prime}\right) \Phi(h)\left(\xi^{\prime}\right)
$$

for some $\xi^{\prime}$ in $X$. So $|f(y)| \leq|\Phi(f)(\xi)|$. By continuity, we see that

$$
|f(y)| \leq|\Phi(f)(\tau(y))| .
$$

If $\Phi(f)(\tau(y))=0$, we can repeat an argument similar to the one in the last paragraph and obtain $f(y)=0$.

Putting all these facts together, we see that the proof of Remark 8 is complete.

Remark 9. $\tau$ is a homeomorphism of $\partial_{\mathcal{A}}(X)$ onto itself.

Proof. We observe first that $\tau$ is injective: if $\tau(x)=\tau(y)$, then $|\Phi(f)(\tau(x))|=$ $|\Phi(f)(\tau(y))|$ and this implies that $|f(x)|=|f(y)|$ for all $f \in \mathcal{A}$ by Remark 8. Since $\mathcal{A}$ separates points of $X$, it is easily seen that there exist functions $f$ such that $f(x)=0, f(y)=1$ proving that $x=y$. Next we show that $\tau$ is continuous. Choose any $x \in X$ and a neighborhood $V$ of $\tau(x)$ and a peaking function $h$ such that

$$
h(\tau(x))=1,|h(y)| \leq 1 / 2 \quad \forall y \in X \backslash V .
$$

$\Phi$ being surjective, there exists a $g$ such that $\Phi(g)=h$. Since $|g| \equiv|\Phi(g(\tau))|$ by Remark 8, if we let $W=\{\xi:|g(\xi)|>1 / 2\}$, then $\tau(W) \subset V$ because if $\xi \in W$, then

$$
|h(\tau(\xi))|=|\Phi(g)(\tau(\xi))|=|g(\xi)|>1 / 2 .
$$

Since $|h(\tau(x))|=|\Phi(g)(\tau(x))|=|g(x)|=1, W$ is a neighborhood of $x$ in $\partial_{\mathcal{A}}(X)$. Thus we have proved that $\tau$ is injective and continuous.

Now since $\Phi$ is a bijection, we see that $\Phi^{-1}$ has the same properties as $\Phi$. Thus there would exist an injective continuous map $\psi: \partial_{\mathcal{A}}(X) \rightarrow \partial_{\mathcal{A}}(X)$ such that

$$
|g(x)| \equiv\left|\Phi^{-1}(g)(\psi(x))\right| \forall x \in \partial_{\mathcal{A}}(X), \forall g \in \mathcal{A} .
$$


Now let $g=\Phi(h)$. Then $|\Phi(h)(x)|=|g(\psi(x))|$. Now let $x=\tau(y)$. Then $|g(y)|=$ $|\Phi(h)(\tau(y))|=|g(\psi(\tau(y)))|$ by Remark 8. Since functions of type $|g|$ separate points of $\partial_{\mathcal{A}}(X)$, we get $\psi(\tau(y)) \equiv y$ and by a similar argument, we also obtain $\tau(\psi(y)) \equiv y$. Thus we proved that $\tau$ is a self-homeomorphism of $\partial_{\mathcal{A}}(X)$.

Remark 10.

$$
f(x)=\Phi(f)(\tau(x)) \text { for all } x \text { in } \partial_{\mathcal{A}}(X) \text { and for all } f \text { in } \mathcal{A} \text {. }
$$

Choose any point $x$ in $\partial_{\mathcal{A}}(X)$. Let $V$ be any open neighborhood of $x$. Since $x$ is in $\partial_{\mathcal{A}}(X)$, there exists a peaking function $h$ such that $h(x)=1$ and the peaking set $P(h)=E$ is contained in $V$. Now by Bishop's theorem 1.5, we can modify $h$ so that it has the same properties as before but, in addition,

$$
|f(z) h(z)|<\max _{E}|f| \text { for all } z \text { outside } E \text {. }
$$

Thus there exists a $\xi$ in $E$ such that $|f(\xi)|=\max _{E}|f|=\|f h\|_{\infty}$. Since $\sigma(f h)=$ $\sigma(\Phi(f) \Phi(h))$, we have $\|f h\|=\|\Phi(f) \Phi(h)\|$ and so there exists a point $z$ such that $f(\xi) h(\xi)=\Phi(f)(z) \Phi(h)(z)$. We may assume that $z \in \partial_{\mathcal{A}}(X)$ since the set where $\Phi(f) \Phi(h)$ assumes the value $f(\xi) h(\xi)$ is a peaking set and every peaking set meets $\partial_{\mathcal{A}}(X)$.

Since $\tau$ is surjective, $z=\tau(\eta)$ for some $\eta$ in $\partial_{\mathcal{A}}(X)$. Now by (2.9) we notice that

$$
|\Phi(f)(\tau(\eta)) \Phi(h)(\tau(\eta))|=|f(\eta) h(\eta)| .
$$

Now $\eta$ must be in $E$ because otherwise $|f(\eta) h(\eta)|<|f(\xi)|$ by (2.11). Thus we have found $\xi, \eta$ in $E$ such that $f(\xi)=\Phi(f)(\tau(\eta))$, since $\Phi(h)(\tau(\eta))=h(\eta)=1$ by Remark 6. Since $\xi, \eta$ lie in $V$ and $V$ is an arbitrary open neighborhood of $x$, we get by continuity of $\tau, f$, and $\Phi(f)$ that $f(x)=\Phi(f)(\tau(x))$. This completes the proof of (2.10).

Remark 11. $\Phi$ is an algebra isomorphism of $\mathcal{A}$ onto itself.

Proof. We already saw that it is a bijection and homogeneous. Let $f, g \in \mathcal{A}$. By (2.10) for any $x$ in $\partial_{\mathcal{A}}(X)$,

$$
f(x)=\Phi(f)(\tau(x)), g(x)=\Phi(g)(\tau(x))
$$

and

$$
f(x) g(x)=\Phi(f g)(\tau(x)), f(x)+g(x)=\Phi(f+g)(\tau(x)) .
$$

Thus

$$
\Phi(f g)(\tau(x))=\Phi(f)(\tau(x)) \Phi(g)(\tau(x)), \Phi(f+g)(\tau(x))=\Phi(f)(\tau(x))+\Phi(g)(\tau(x)) .
$$

Since $\tau$ is surjective, we get

$$
\Phi(f)(x) \Phi(g)(x)=\Phi(f g)(x), \Phi(f+g)(x)=\Phi(f)(x)+\Phi(g)(x)
$$

on all of $\partial_{\mathcal{A}}(X)$ and then by the maximum principle on all of $X$. This completes the proof of Remark 11. The algebraic isomorphism $\Phi: \mathcal{A} \rightarrow \mathcal{A}$ gives rise to a weak* homeomorphism $\Phi^{*}: \mathcal{A}^{*} \rightarrow \mathcal{A}^{*}$, which in turn induces a homeomorphism $\Lambda$ of $X$ (the maximal ideal space of $\mathcal{A}$ ) onto itself and hence we can state

Remark 12. There exists a self-homeomorphism $\Lambda$ of $X$ onto itself such that

$$
\Phi(f)(\Lambda(x))=f(x) \text { on all of } X .
$$

But in view of (2.10), we see that $\Lambda(x)=\tau(x)$ for all $x$ in $\partial_{\mathcal{A}}(X)$. This completes the proof of the Main Theorem announced in the abstract. 
Conclusion. We conclude this paper by observing:

If $X$ is a compact Hausdorff space (not necessarily first countable), then our Main Theorem clearly holds for $C_{\mathbb{R}(X)}$ - the Choquet boundary being $X$ and the peaking functions being those given by Urysohn's lemma - and it follows that Theorem 6 in [M] is valid in this general setting with the same proof as given there.

\section{ACKNOWLEDGMENTS}

The second author would like to thank the Department of Mathematics of the University of Toledo for its hospitality during the period this paper was written, and both authors thank the referee for his kind comments.

\section{REFERENCES}

[M] L. Molnár, Some Characterizations of the Automorphisms of $B(H)$ and $C(X)$, Proceedings of the American Mathematical Society 130, no.1 (2002), 111-120. MR 1855627 (2002m:47047)

[Br] A. Browder, Introduction to Function Algebras, W. A. Benjamin, Inc., 1969. MR0246125 $(39: 7431)$

[P] R. Phelps, Lectures on Choquet's theorem, D. Van Nostrand Company Inc., Princeton, 1966. MR0193470 (33:1690)

[BL] E. Bishop and K. de Leeuw, The representations of linear functionals by measures on sets of extreme points, Ann. Inst. Fourier (Grenoble) 9 (1959), 305-331. MR0114118 (22:4945)

Department of Mathematics, University of Toledo, Toledo, Ohio 43606

E-mail address: rnagise@math.utoledo.edu

Indian Statistical Institute-Calcutta, Statistics and Mathematics Unit, 203 B.T. Road, Calcutta 700 108, India

E-mail address: ashoke@isical.ac.in 